Oskar Luger, Astrid Tröstl, Katrin Urferer

Über Gentechnik und Klone 
Lebensformen

herausgegeben von

Ulrich Beer $(\dagger)$ und Roswitha Stemmer-Beer

Band 65 
Oskar Luger, Astrid Tröstl, Katrin Urferer

\title{
Über Gentechnik und Klone
}

\author{
Eine Übersicht
}

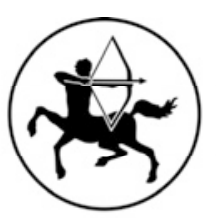

Centaurus Verlag \& Media UG 


\section{Über die Autoren}

Dr. Oskar Luger, geboren 1944, hat an der Universität Wien Biologie studiert und war anschließend mehrere Jahre in der biologisch-medizinischen Grundlagenforschung tätig. Bis zu seiner Pensionierung war er am Erzbischöflichen Gymnasium in Hollabrunn bei Wien als Lehrer tätig. Oskar Luger hat zahlreiche Artikel zu Gentechnik, Stammzellen und Evolution veröffentlicht. und ist seit über 20 Jahren in der Erwachsenenbildung zum Thema tätig.

Mag. Astrid Tröstl hat an der Universität Wien Biologie und Umweltkunde und Geographie und Wirtschaftskunde auf Lehramt studiert und ist seither am Erzbischöflichen Gymnasium Hollabrunn als Lehrerin tätig. Sie hat durch ihre langjährige Mitarbeit bei der ARGE Nikotininstitut zahlreiche Artikel zum Themenkreis Rauchen in medizinischen Zeitschriften veröffentlicht.

Mag. Katrin Urferer hat an der Universität Wien Biologie und Umweltkunde und Mathematik auf Lehramt studiert und ist seither am Erzbischöflichen Gymnasium Hollabrunn als Lehrerin tätig.

\section{Bibliografische Informationen der Deutschen Nationalbibliothek}

Die Deutsche Nationalbibliothek verzeichnet diese Publikation in der Deutschen Nationalbibliografie; detaillierte bibliografische Daten sind im Internet über http://dnb.d-nb.de abrufbar.

Gedruckt auf säurefreiem und chlorfrei gebleichtem Papier.

ISBN 978-3-86226-201-4 ISBN 978-3-86226-271-7 (eBook) DOI 10.1007/978-3-86226-271-7

\section{ISSN 1612-2739}

Alle Rechte, insbesondere das Recht der Vervielfältigung und Verbreitung sowie der Übersetzung, vorbehalten. Kein Teil des Werkes darf in irgendeiner Form (durch Fotokopie, Mikrofilm oder ein anderes Verfahren) ohne schriftliche Genehmigung des Verlages reproduziert oder unter Verwendung elektronischer Systeme verarbeitet, vervielfältigt oder verbreitet werden.

(C) CENTAURUS Verlag \& Media KG, Freiburg 2012

www.centaurus-verlag.de

Umschlaggestaltung: Jasmin Morgenthaler, Visuelle Kommunikation

Umschlagsabbildung: Julia Herzog

Satz: Vorlage der Autoren 


\section{Vorwort}

Es gibt wohl keinen anderen Bereich von Wissenschaft und Technik, der so umfassend und grundlegend in unser Leben eingreift, wie die Gentechnologie.

Betroffen ist vor allem die Ernährung. Außerhalb Europas breiten sich gentechnisch veränderte Pflanzen immer weiter aus, auch wenn es Widerstand dagegen gibt. Die großen Chemie- und Saatgutkonzerne bekommen über Patente für gentechnisch verändertes Saatgut zunehmend Kontrolle über den Pflanzenbau.

Zunehmenden Einfluss hat die Gentechnik auf die Medizin, seien es Medikamente wie Insulin für Diabetikerinnen und Diabetiker oder seien es Gentests, die zu einer zunehmenden Überwachung der Schwangerschaft führen oder Krankheitsrisiken vorhersagen sollen.

Um unseren Schülerinnen und Schülern ein umfassenderes Wissen auf den Weg mitzugeben, haben wir vor Jahren ein schmales Skriptum zu den häufigsten Anwendungsgebieten der Gentechnik und ihren Folgen verfasst. Dieser ursprünglich bescheidene Lehrbehelf ist durch Aktualisierung, Erweiterung und Ergänzung zu allgemein bedeutsamen Themen und den Bereich des Klonens zu diesem Buch geworden.

Es soll eine auch ohne großes biologisches Vorwissen gut verständliche, überschaubare Einführung in jene Bereiche der Gentechnik geben, von der wir mehr oder weniger betroffen sind. Ausgenommen sind z. B. die Anwendungen in der biomedizinischen Forschung. Das wäre eine andere, sehr umfangreiche Geschichte, denn nicht nur in der angewandten, sondern auch in der Grundlagenforschung spielt die Gentechnologie eine immer wichtigere Rolle.

Es erscheint uns wichtig, dass ein Mensch Bescheid weiß über etwas, das ihn betrifft. So hoffen wir, dass dieses kleine Buch seinen Beitrag leisten kann, der Gentechnologie nicht mit Kopfschütteln gegenüber zu stehen, sondern Einsicht und damit Beurteilungsfähigkeit zu bekommen.

Der Objektivität wegen haben wir die reinen biologischen Fakten, das „Wie“ und offizielle „Warum“ gentechnischer Manipulationen von den Probleme, die daraus erwachsen oder erwachsen können und unserer eigenen persönlichen Meinung getrennt

Wir möchten zum Abschluss noch danke sagen - dem Centaurus-Verlag und da vor allem Frau Petra Sanft und Jens Benicke für die freundliche und aufmunternde Betreuung - Julia Herzog für das Titelphoto - Hermann Rainer für stilistischen Rat und Martina Luger für viele anregende Diskussionen in der Entstehung das Textes. 


\section{Inhaltsverzeichnis}

$\begin{array}{ll}\text { Vorwort } & 5\end{array}$

$\begin{array}{ll}\text { Inhaltsverzeichnis } & 7\end{array}$

$\begin{array}{lr}\text { Einleitung und Vorbemerkungen } & 9\end{array}$

$\begin{array}{ll}\text { Überblick über Gentechnologie } & 11\end{array}$

1 Gentechnisch veränderte Pflanzen 13

1.1 Resistenz gegen Herbizide (Unkrautvernichter) 15

1.2 Resistenz gegen Insekten 15

1.3 Gentechnisch veränderte Pflanzen mit Zusatznutzen

1.4 Die Terminatortechnologie 28

1.5 Industriell oder energetisch interessante Pflanzen 29

$\begin{array}{lll}1.6 & \text { Pharmapflanzen } & 30\end{array}$

1.7 Gentechnisch veränderte Pflanzen als Futtermittel 31

2 Allgemeine Probleme der Gentechnik 33

2.1 Die Patentierung 33

2.2 Biopiraterie $\quad 35$

2.3 Genetische Erosion $\quad 36$

2.4 Koexistenz zwischen gentechnischer und gentechnikfreier Landwirtschaft $\quad 37$

2.5 Unerwartete Nebeneffekte 40

$\begin{array}{lll}2.6 & \text { Allergierisiko } & 40\end{array}$

2.7 Hungerproblematik 41

2.8 Gentechnische Veränderung versus Züchtung 42

3 Gentechnisch veränderte Tiere 43

3.1 Gentechnisch veränderte Tiere in der medizinischen Anwendung 44

4 Lebensmittelzusätze aus gentechnisch veränderten Mikroorganismen 47

4.1 Medizinische Anwendung am Beispiel der Medikamentenproduktion 48

4.2 Schlussüberlegungen $\quad 53$ 
5 Gentherapie $\quad 55$

5.1 Somatische Gentherapie 55

5.2 Keimbahntherapie $\quad 56$

$\begin{array}{lll}5.3 & \text { Gendiagnose } & 57\end{array}$

5.3.1 Monogen vererbte Krankheiten $\quad 58$

5.3.2 Polygen vererbte Krankheiten und die Umwelt $\quad 59$

5.3.3 Das Recht auf Nichtwissen $\quad 62$

5.3.4 ArbeitgeberInnen und Gendiagnosen $\quad 62$

5.3.5 Krankenkassen $\quad 62$

5.3.6 Datentransfer 63

5.3.7 Pränataldiagnosen $\quad 63$

5.4 Die Präimplantationsdiagnostik (PID) 66

$\begin{array}{lll}5.5 & \text { Schlussbemerkungen } & 68\end{array}$

6 Von Klonen und Menschen $\quad 71$

6.1 Gurdons Kerntransplantationen mit Krallenfröschen 71

$\begin{array}{lll}6.2 & \text { Dolly } & 72\end{array}$

6.3 Menschliche Klone $\quad 72$

6.4 Reproduktives und therapeutisches Klonen 73

6.4.1 Embryonale und adulte Stammzellen $\quad 73$

$\begin{array}{ll}\text { 6.4.2 Induzierte pluripotente Stammzellen } & 75\end{array}$

$\begin{array}{ll}\text { 6.4.3 Chimären } & 76\end{array}$

6.5 Abschließende Gedanken 76

Tabellenverzeichnis $\quad 79$

$\begin{array}{lr}\text { Weiterführende und vertiefende Literatur } & \mathbf{8 0}\end{array}$

Risiken, Bedenken und persönliche Anmerkungen der Autoren wurden grau hinterlegt. 